\title{
Generalized Whitney partitions
}

by

Michał Rams (Warszawa)

\begin{abstract}
We prove that the upper Minkowski dimension of a compact set $\Lambda$ is equal to the convergence exponent of any packing of the complement of $\Lambda$ with polyhedra of size not smaller than a constant multiple of their distance from $\Lambda$.
\end{abstract}

1. Results. For a set $K \subset \mathbb{R}^{d}$ denote by $B_{r}(K)$ its $r$-neighbourhood. We define the upper Minkowski dimension as

$$
\operatorname{dim}(K)=d+\limsup _{r \rightarrow 0} \frac{\log \operatorname{vol} B_{r}(K)}{-\log r}
$$

Another, more common definition of this dimension is as follows: let $N_{r}(K)$ be the minimal number of sets in a covering of $K$ with balls of radius $r$. Then

$$
\operatorname{dim}(K)=\limsup _{r \rightarrow 0} \frac{\log N_{r}(K)}{-\log r} .
$$

Note that

$$
\operatorname{dim}(K)=\operatorname{dim}(\bar{K}) .
$$

For other properties of this notion, see [6] or [4].

Let $\Lambda$ be a bounded subset of $\mathbb{R}^{d}$ with closure of volume 0 . Let $\left(E_{i}\right)$ be a family of convex closed $d$-dimensional polyhedra in $\mathbb{R}^{d}$, disjoint from $\Lambda$, covering the complement of $\bar{\Lambda}$ (to be denoted by $\bar{\Lambda}^{\mathrm{c}}$ ) and with pairwise disjoint interiors. We will also need these polyhedra to have uniformly bounded ratio of their external to internal radii. By the external radius of a set we mean the smallest radius of a ball containing it; similarly, the internal radius of a set is the greatest radius of a ball contained in it. Such families of polyhedra will be called uniformly regular.

We will also demand that the edges of these polyhedra are long, i.e. have lengths uniformly comparable to the diameter of the polyhedron. As

2000 Mathematics Subject Classification: 05B40, 52C17.

Research supported by Polish KBN Grant No 2 P03A 02512. 
an example we can take the family of maximal dyadic cubes disjoint from $\Lambda$. The diameter of $E_{i}$ will be denoted by $\left|E_{i}\right| . \Lambda$ will be called the residual set of the family $\left(E_{i}\right)$.

There are two conditions which are usually imposed on such families:

(i) $\left|E_{i}\right| \geq c \operatorname{dist}\left(E_{i}, \Lambda\right)$,

(ii) $\left|E_{i}\right| \leq c \operatorname{dist}\left(E_{i}, \Lambda\right)$.

If a family $\left(E_{i}\right)$ satisfies both the conditions (i) and (ii), it is called a Whitney family. A family which satisfies only (i) will be called a generalized Whitney family. See [10] for some properties of Whitney families of cubes.

For a family $\left(E_{i}\right)$ one can consider the convergence exponent of $\left(E_{i}\right)$, defined as follows:

$$
s_{E}=\inf \left\{t: \sum_{i=1}^{\infty}\left|E_{i}\right|^{t}<\infty\right\},
$$

where the sum is taken over $E_{i}$ with diameter smaller than any given constant. This notion is closely related to the notion of Poincaré exponent (see [7] or [2]).

It is well known (see for example [5] or [1]) that for any Whitney family

$$
s_{E}=\operatorname{dim}(\Lambda),
$$

where $\Lambda$ is the residual set for $\left(E_{i}\right)$.

Our result states that the same is true for generalized Whitney families.

THEOREM 1.1. Let $\Lambda$ be a bounded subset of $\mathbb{R}^{d}$ with closure of zero d-dimensional measure. Let $\left(E_{i}\right)$ be any generalized Whitney family of uniformly regular convex polyhedra with long edges in $\mathbb{R}^{d}$, for which $\Lambda$ is a residual set, and let $s_{E}$ be its convergence exponent. Then

$$
s_{E}=\operatorname{dim}(\Lambda) .
$$

For a special case this result was obtained (by a different method) by Tricot [13]. See also [11] and [12] for related results.

The results of this paper are part of my $\mathrm{PhD}$ thesis [9].

The rest of the paper is divided as follows. Section 2 contains some basic information, notations and motivations for the problem. Section 3 contains the proof of Theorem 1.1 in the special case when the family $\left(E_{i}\right)$ consists only of cubes, with edges parallel to the coordinate axes - this is the case done in [13]. Section 4 contains the proof of Theorem 1.1 in full generality.

Remark on notation: $c$ stands for any constant, not necessarily the same at each occurrence. If a constant has to be chosen depending on some variables, it is denoted like $c(t, u, v)$. The dependence on $d$ is omitted. The notation $f \approx g$ means the existence of two constants $c_{1}, c_{2}$, depending only on $d$, such that $c_{1} f \leq g \leq c_{2} f$. The set of natural numbers contains zero. 
I want to thank the referee for many extremely helpful suggestions. Among other things, he drew my attention to Tricot's thesis [13]. In the original version, I worked only with compact $\Lambda$ so the elements $E_{i}$ of partitions were always at a positive distance from $\Lambda$-it was also the referee who suggested this constraint could be omitted.

2. Introduction. We recall some notations. In what follows $\Lambda$ is a bounded subset of $\mathbb{R}^{d}$ with closure of $d$-dimensional volume $0,\left(E_{i}\right)$ is a generalized Whitney family for $\Lambda$, and $s_{E}$ denotes the convergence exponent of the family $\left(E_{i}\right)$. One easily sees that $s_{E} \in[0, d]$.

As the definition of $s_{E}$ depends only on small sets $E_{i}$, we can freely remove from this family all the sets with diameters greater than any given constant. Let us choose this constant to be greater than the diameter of all the $E_{i}$ intersecting $\bar{\Lambda}$, so the family will still cover some neighbourhood of $\bar{\Lambda}$.

Let us start from a simple fact that was our motivation for studying generalized Whitney partitions and their convergence exponents. Let $\left(E_{i}\right)$ be the dyadic covering of $\Lambda^{\mathrm{c}}$, i.e. the covering of $\Lambda^{\mathrm{c}}$ with maximal cubes of the form $\left[k_{1} / 2^{l},\left(k_{1}+1\right) / 2^{l}\right] \times \ldots \times\left[k_{d} / 2^{l},\left(k_{d}+1\right) / 2^{l}\right]$, disjoint from $\Lambda$. This partition is generalized Whitney, but not necessarily Whitney.

Proposition 2.1. For the dyadic partition the assertion of Theorem 1.1 holds.

Proof. We can assume that $\Lambda \subset I=(-1,1)^{d}$ and restrict the family $\left(E_{i}\right)$ to subsets of $\bar{I}$.

We denote by $D(n)$ the number of dyadic cubes with edges of length $2^{-n}$ belonging to $\left(E_{i}\right)$. Let $A(n)$ denote the number of dyadic cubes with edges of length $2^{-n}$, intersecting $\Lambda$. We also write $G_{t}(n)=A(n) \cdot 2^{-n t}$.

From the construction of the dyadic partition we can write

$$
D(n+1)=2^{d} A(n)-A(n+1) .
$$

The definition of upper Minkowski dimension can be written as follows:

$$
\operatorname{dim}(\Lambda)=\limsup \frac{1}{n} \log _{2} A(n) .
$$

Hence

$$
\begin{array}{rr}
\forall t>\operatorname{dim}(\Lambda) \exists c(t) & A(n)<c(t) \cdot 2^{n t}, \\
\forall t<\operatorname{dim}(\Lambda) \exists c(t) \exists\left(n_{i}\right)_{i=1}^{\infty} & A\left(n_{i}\right)>c(t) \cdot 2^{n_{i} t} .
\end{array}
$$

Let us look at the series

$$
H(t)=\sum_{i=1}^{\infty} D(i) \cdot 2^{-i t}=\sum_{i=1}^{\infty}\left(2^{d-t} G_{t}(i-1)-G_{t}(i)\right) .
$$


We need to prove that it converges for $t>\operatorname{dim}(\Lambda)$ and diverges for $t<$ $\operatorname{dim}(\Lambda)$. The first assertion is immediate, since for $t>\operatorname{dim}(\Lambda)$ the series $\sum G_{t}(i)$ is convergent. In what follows we assume $t<\operatorname{dim}(\Lambda)$.

We have (from (1)) a sequence $\left(n_{i}\right)$ of natural numbers for which $G_{t}\left(n_{i}\right)$ $>1$ (even $\left.G_{t}\left(n_{i}\right) \nearrow \infty\right)$. The sequence $G_{d}\left(n_{i}\right)$ is a subsequence of $G_{d}(n)$, so it decreases to 0 . We can assume (passing to a subsequence if necessary) that $G_{d}\left(n_{i}\right)>2 G_{d}\left(n_{i+1}\right)$ for all $i$. Then

$$
H(t) \geq \sum_{i=1}^{\infty} \sum_{k=n_{i}+1}^{n_{i+1}} D(k) \cdot 2^{-k t}=\sum_{i=1}^{\infty} H_{i}(t),
$$

where

$$
H_{i}(t)=\sum_{k=n_{i}+1}^{n_{i+1}} D(k) \cdot 2^{-k t} .
$$

We have

$$
\begin{aligned}
& G_{d}\left(n_{i}\right)-G_{d}\left(n_{i+1}\right)=\sum_{k=n_{i}+1}^{n_{i+1}} D(k) \cdot 2^{-k d} \leq 2^{-\left(n_{i}+1\right)(d-t)} H_{i}(t), \\
& G_{d}\left(n_{i}\right)-G_{d}\left(n_{i+1}\right) \geq \frac{1}{2} G_{d}\left(n_{i}\right)=2^{-n_{i}(d-t)-1} G_{t}\left(n_{i}\right) .
\end{aligned}
$$

Finally,

$$
H_{i}(t) \geq 2^{d-t-1} G_{t}\left(n_{i}\right)>2^{d-t-1}
$$

so the series $H(t)$ diverges.

Now we proceed to the general case. We denote by $K_{n}$ the union of $\bar{\Lambda}$ and all the sets $E_{i}$ with diameters smaller than $2^{-n}$; this set is compact and contains $\Lambda$. We can estimate

$$
\sum\left|E_{i}\right|^{t} \approx \sum_{n=0}^{\infty} \frac{\operatorname{vol}\left(K_{n} \backslash K_{n+1}\right)}{2^{-n d}} \cdot 2^{-n t} .
$$

The volumes of the sets $K_{n}$ decrease to zero, hence this series is equal to

$$
\begin{array}{r}
\sum_{n=0}^{\infty}\left(\operatorname{vol} K_{n}-\operatorname{vol} K_{n+1}\right)+\sum_{n=1}^{\infty} \sum_{m=n}^{\infty}\left(2^{n(d-t)}-2^{(n-1)(d-t)}\right)\left(\operatorname{vol} K_{m}-\operatorname{vol} K_{m+1}\right) \\
=\operatorname{vol} K_{0}+\left(1-2^{t-d}\right) \sum_{n=1}^{\infty} 2^{n(d-t)} \operatorname{vol} K_{n} .
\end{array}
$$

Hence

$$
s_{E}=d+\limsup \frac{1}{n} \log _{2}\left(\operatorname{vol} K_{n}\right) .
$$

Let $\delta$ be a constant, to be chosen afterwards. We denote by $L_{n}$ the $\delta_{n}$-neighbourhood of $\Lambda$, where $\delta_{n}=\delta \cdot 2^{-n}$. Independently of the choice of 
$\delta$ we can rewrite the definition of upper Minkowski dimension as follows:

$$
\operatorname{dim}(\Lambda)=d+\limsup \frac{1}{n} \log _{2}\left(\operatorname{vol} L_{n}\right) .
$$

If we choose $\delta$ sufficiently large then $K_{n} \subset L_{n}$. On the other hand, if $\left(E_{i}\right)$ is a Whitney partition (not only generalized Whitney) then for $\delta$ small enough we have $K_{n} \supset L_{n}$. So the following lemma follows immediately from (2) and (3):

LEMma 2.2. We have

$$
s_{E} \leq \operatorname{dim}(\Lambda) .
$$

If $\left(E_{i}\right)$ is a Whitney partition then we have equality.

Hence in the case $\operatorname{dim}(\Lambda)=0$ the assertion of Theorem 1.1 is immediately true. In the rest of the paper we assume $\operatorname{dim}(\Lambda)$ to be positive.

3. Cubes. In this section we prove Theorem 1.1 in a special case. Throughout this section we demand all the sets $E_{i}$ to be cubes with edges parallel to coordinate axes. The proof in the general case is similar; we present the simpler case first to outline our approach.

Let us begin with two lemmas that allow us to restrict our attention to the case of $\Lambda$ of small dimension. We denote by $l$ the greatest integer strictly smaller than $\operatorname{dim}(\Lambda)$ - it is in $[0, d-1]$.

LEMma 3.1. $\operatorname{dim}(\Lambda)>d-1 \Rightarrow s_{E}=\operatorname{dim}(\Lambda)$.

Proof. We construct a new family $\left(E_{i j}^{\prime}\right)$ by applying the following algorithm to all the cubes $E_{i}$. First we divide the cube into $2^{d}$ smaller ones. In the second step we divide all of them again. In all the next steps we divide not all of them, but only those which touch the boundary of the initial cube $E_{i}$. We execute these steps infinitely many times. Figure 1 shows the situation after a few steps.

Our new family has residual set $\Lambda^{\prime}$ greater than $\Lambda$-it also contains the boundaries of all the cubes $E_{i}$-and this family is a Whitney family. We then know that

$$
s_{E^{\prime}}=\operatorname{dim}\left(\Lambda^{\prime}\right) \geq \operatorname{dim}(\Lambda) \geq s_{E}
$$

where $s_{E^{\prime}}$ stands for the convergence exponent of the resulting family $\left(E_{i j}^{\prime}\right)$.

Let us look at a cube $E_{i}$ from the initial family. It is divided into an infinite number of cubes $E_{i j}^{\prime}$; one can check that there are no more than $c \cdot 2^{-n(d-1)}$ cubes of side length $2^{-n}\left|E_{i}\right|$ in it. So, for any $t \in(d-1, d]$ we can write

$$
\left|E_{i}\right|^{t} \leq \sum_{E_{i j}^{\prime} \subset E_{i}}\left|E_{i j}^{\prime}\right|^{t}<c(t)\left|E_{i}\right|^{t}
$$




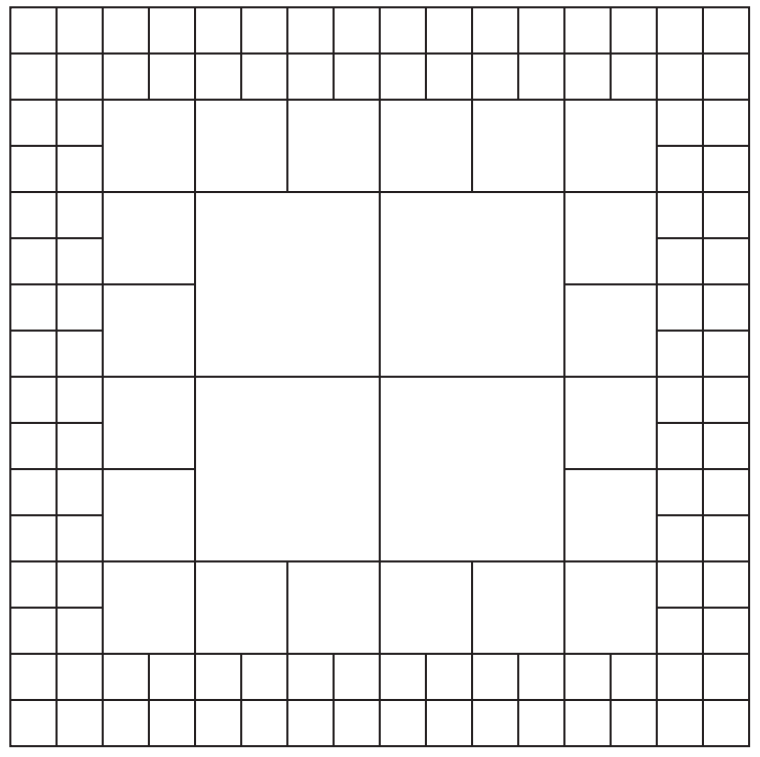

Fig. 1. The subpartition $E_{i j}^{\prime}$ after the fourth step

Hence for any such $t$ the series $\sum\left|E_{i}\right|^{t}$ and $\sum\left|E_{i j}^{\prime}\right|^{t}$ are simultaneously convergent or divergent. The latter is convergent when $t>s_{E^{\prime}}$ and divergent when $t<s_{E^{\prime}}$. As $s_{E^{\prime}} \in(d-1, d]$, the same must be true for the former, hence the convergence exponents of the old and new families have to be the same. This, together with the estimate on $s_{E^{\prime}}$ and Lemma 2.2, gives the assertion of the lemma.

This argument will reappear later in more complicated versions.

The next lemma is only a slight strengthening of the previous one.

LEMMA 3.2. If some projection of $\bar{\Lambda}$ onto a $(d-1)$-dimensional hyperplane has positive $(d-1)$-dimensional measure then $s_{E}=\operatorname{dim}(\Lambda)$.

Proof. Let $\Pi$ be such a projection. Then

$$
\sum_{E_{i}}\left|E_{i}\right|^{d-1} \geq \sum_{E_{i}} \operatorname{vol}_{d-1}\left(\Pi\left(E_{i}\right)\right) \geq \int_{\Pi(\bar{\Lambda})} n(x) d x^{d-1},
$$

where $n(x)$ is the number of cubes $E_{i}$ whose projection contains the point $x$. Of course, $n(x)=\infty$ everywhere on $\Pi(\Lambda)$ so the integral is infinite. This means that $s_{E} \geq d-1$. As the projection can never increase the dimension, the upper Minkowski dimension of $\Lambda$ has to be no smaller than $d-1$. If it equals $d-1$ then by Lemma 2.2 the convergence exponent also has to be $d-1$. And the case $\operatorname{dim}(\Lambda)>d-1$ was dealt with in Lemma 3.1. 
For the rest of the section we can thus assume $l \leq d-2$.

Now we define some additional notations, to be used in the remainder of the paper. For any $n$ we can choose a set of points from $\Lambda$ in such a way that the cubes of side length $2 \delta_{n}$ with centres at the chosen points and with sides parallel to coordinate axes are pairwise disjoint. We call the maximal such set $M_{n}$, and the cube as above with centre at $x \in M_{n}$ will be denoted by $B_{n}(x)$. One can check that

$$
\sharp M_{n} \approx 2^{n d} \operatorname{vol} L_{n} .
$$

Let $n_{j}$ be a sequence of natural numbers such that

$$
\operatorname{dim}(\Lambda)=d+\lim \frac{1}{n_{j}} \log _{2}\left(\operatorname{vol} L_{n_{j}}\right) .
$$

We then have for any positive $\varepsilon^{\prime}$ the estimates

$$
\begin{gathered}
\sharp M_{n_{j}} \geq c\left(\varepsilon^{\prime}\right) \delta_{n_{j}}^{\operatorname{dim}(\Lambda)-\varepsilon^{\prime}}, \\
\operatorname{vol} L_{n} \leq c \sharp M_{n} \delta_{n}^{d} .
\end{gathered}
$$

We prove Theorem 1.1 by contradiction. Throughout the rest of the section we assume that the convergence exponent for $\left(E_{i}\right)$ is not equal to (hence strictly smaller than) the upper Minkowski dimension of $\Lambda$.

We postpone the proof of the following key lemma until the end of the section.

Lemma 3.3. There exists $C$ such that for any $\varepsilon$ and any $n_{j}$ sufficiently large at least $\frac{1}{2} \sharp M_{n}$ of the sets $B_{n_{j}}(x) \cap K_{n_{j}}$ have volume smaller than $\varepsilon \delta_{n_{j}}^{d}$ and each of these contains an $(l+1)$-dimensional cube $D(x)$ of side length $C \cdot \delta_{n_{j}}$ lying at a distance at least $C \cdot \delta_{n_{j}}$ from the boundary of $B_{n_{j}}(x)$.

From this lemma Theorem 1.1 easily follows. As the intersection of the set $B_{n_{j}}(x)$ with the interior of $K_{n_{j}}$ is open, there is a whole rectangular parallelepiped in it, say $D^{\prime}(x)$, one of whose $(l+1)$-dimensional sides is $D(x)$.

Now consider only those cubes $E_{i}$ which meet $D^{\prime}(x)$ in such a way that the projection $\Pi$ of $E_{i}$ onto the $(l+1)$-dimensional surface passing through $D(x)$ lies inside $D(x)$; those cubes lie in $B_{n_{j}}(x) \cap K_{n_{j}}$, so their edges are not greater than $\varepsilon^{1 / d} \delta_{n_{j}}$. Their projections cover $D(x)$ except possibly a strip of width $\varepsilon^{1 / d} \delta_{n_{j}}$ at all borders, and except a set of $(l+1)$-dimensional measure 0 elsewhere. As we can take $\varepsilon$ arbitrarily small in Lemma 3.3, we can guarantee that the $(l+1)$-dimensional volume of the union of those projections is greater than $c \delta_{n_{j}}^{l+1}$.

For $t<l+1$ the ratio of $\left|E_{i}\right|^{t}$ to the volume of the projection of $E_{i}$ onto $D(x)$ (which lies between $\left|E_{i}\right|^{l+1}$ and $c \cdot\left|E_{i}\right|^{l+1}$ ) is smallest when $\left|E_{i}\right|$ 
is greatest. Hence

$$
\begin{aligned}
\sum_{E_{i} \subset B_{n_{j}}(x)}\left|E_{i}\right|^{t} & \geq \operatorname{vol}_{l+1}(D(x)) \inf \frac{\left|E_{i}\right|^{t}}{\operatorname{vol}_{l+1}\left(\Pi\left(E_{i}\right)\right)} \\
& \approx c \delta_{n_{j}}^{l+1}\left(\varepsilon^{1 / d} \delta_{n_{j}}\right)^{t-l-1}=c(t, \varepsilon) \delta_{n_{j}}^{t} .
\end{aligned}
$$

This is the sum in one of the cubes $B_{n_{j}}(x)$ only-to estimate this sum over all the cubes $E_{i}$ we should multiply it by the number of such cubes $B_{n_{j}}(x)$, that is, by $\frac{1}{2} \sharp M_{n_{j}}$ (Lemma 3.3). Take any $\varepsilon^{\prime}>0$. Set

$$
t=\operatorname{dim}(\Lambda)-2 \varepsilon^{\prime}<l+1 \text {. }
$$

Using (5) we get an estimate for the total sum:

$$
\sum\left|E_{i}\right|^{t} \geq c\left(\varepsilon, \varepsilon^{\prime}\right) \cdot \delta_{n_{j}}^{-\varepsilon^{\prime}},
$$

and this can be arbitrarily large for we can take $n_{j}$ arbitrarily large. Hence $s_{E} \geq \operatorname{dim}(\Lambda)-2 \varepsilon^{\prime}$, which (together with Lemma 2.2) ends the proof of Theorem 1.1.

Now we prove Lemma 3.3. We still assume that $s_{E}<\operatorname{dim}(\Lambda)$.

Let us see what happens in cubes $B_{n_{j}}(x)$ with centres in $M_{n_{j}}$. If for infinitely many $n_{j}$,

$$
\frac{\operatorname{vol}\left(K_{n_{j}} \cap L_{n_{j}}\right)}{\operatorname{vol} L_{n_{j}}} \geq c>0,
$$

then the measure of $K_{n_{j}}$ would be at least proportional to one of $L_{n_{j}}$, hence we would get $s_{E} \geq \operatorname{dim}(\Lambda)$ - a contradiction.

For the rest of this section we assume that this fraction goes to 0 as $n_{j}$ goes to the infinity. Then for any $\varepsilon>0$, for all $n_{j}$ large enough and for $c$ taken from (5),

$$
\frac{\operatorname{vol}\left(K_{n_{j}} \cap L_{n_{j}}\right)}{\operatorname{vol} L_{n_{j}}} \leq \frac{\varepsilon}{2 c} .
$$

This means that for any $\varepsilon>0$ and all $n_{j}$ large enough the inequality

$$
\frac{\operatorname{vol}\left(B_{n_{j}}(x) \cap K_{n_{j}}\right)}{\operatorname{vol} B_{n_{j}}(x)}<\varepsilon
$$

holds for at least half the points in $M_{n_{j}}$, because the volume of any $B_{n}(x)$ is the same.

We now consider for a while a new family $\left(E_{i k}^{\prime}\right)$, which is a subpartition of $\left(E_{i}\right)$, similar to the one in the proof of Lemma 3.1. Like there, we first divide a cube $E_{i}$ into $4^{d}$ cubes, but then we divide not all those which touch the boundary of $E_{i}$ but only those which touch one of its $l$-dimensional sides. See Figure 2. 


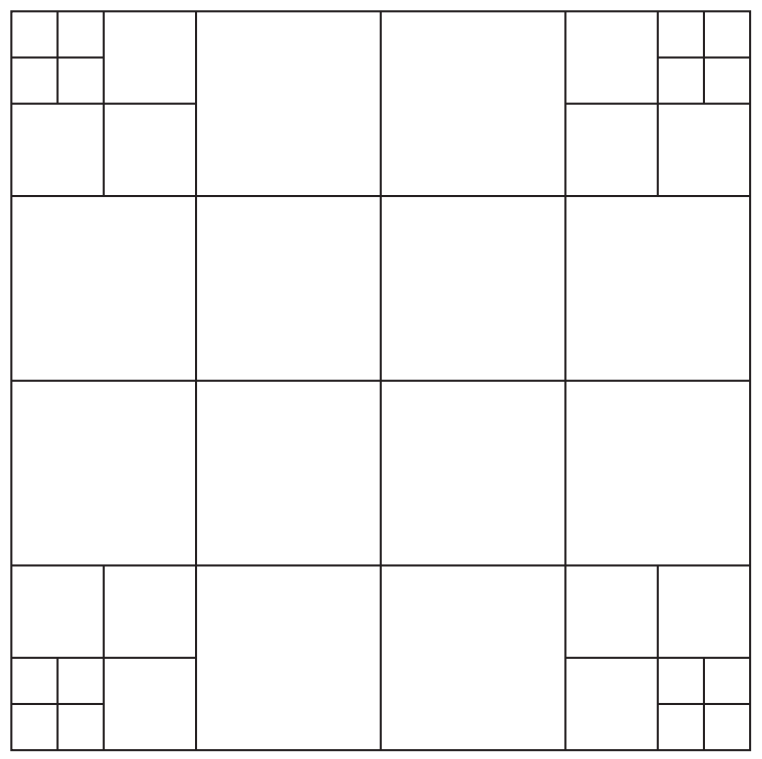

Fig. 2. The subpartition $E_{i k}^{\prime}$ for $d=2, l=0$ after the fourth step

The construction from Lemma 3.1 was a special case $l=d-1$. Like there, for any $t>l$ we have

$$
\sum_{E_{i k}^{\prime} \subset E_{i}}\left|E_{i k}^{\prime}\right|^{t} \approx c(t)\left|E_{i}\right|^{t}
$$

(because this family is a Whitney partition for the union of $l$-dimensional sides of $E_{i}$ ).

Hence

$$
s_{E}<\operatorname{dim}(\Lambda) \Leftrightarrow s_{E^{\prime}}<\operatorname{dim}(\Lambda) .
$$

Then we see that even for these new, smaller cubes the set $K_{n_{j}}^{\prime}$ has small intersection with most of the cubes $B_{n_{j}}(x)$ for $n_{j}$ large enough, that is, the inequality (7) also holds for $K_{n_{j}}^{\prime}$.

Let us now look at the situation in one of those cubes $B_{n_{j}}(x)$ for which this intersection is small. The intersection is the whole $B_{n_{j}}(x)$ except those cubes $E_{i k}^{\prime}$ which are too big. From the very definition of $E_{i k}^{\prime}$, the side length of $E_{i k}^{\prime}$ is roughly proportional to the distance between this cube and the closest $l$-dimensional side of the cube $E_{i}$ it lies in. So, if a cube $E_{i k}^{\prime}$ meets $B_{n_{j}}(x)$ and is too big to be in $K_{n_{j}}$ then no $l$-dimensional sides of the cube $E_{i}$ can meet $B_{n_{j}}(x)$ provided $\delta$ is sufficiently small. As the corresponding sides of $E_{i}$ and $B_{n_{j}}(x)$ are parallel, $E_{i}$ has to contain one of the $(l+1)$-dimensional sides of $B_{n_{j}}(x)$.

We can now return to the family $\left(E_{i}\right)$. We have just proved the following statement: 
For any $\varepsilon$ and for $n_{j}$ large enough, for at least half the cubes $B_{n_{j}}(x)$ the intersection $B_{n_{j}}(x) \cap K_{n_{j}}^{\mathrm{c}}$ has volume at least $(1-\varepsilon) \operatorname{vol}\left(B_{n_{j}}(x)\right)$. This intersection is a union of intersections of $B_{n_{j}}(x)$ with large cubes $E_{i}$ (those of side length greater than $\left.\frac{1}{\delta}\left|B_{n_{j}}(x)\right|\right)$-but the intersections with cubes $E_{i}$ containing less than $2^{l+1}$ vertices of $B_{n_{j}}(x)$ have total volume not greater than $\varepsilon \operatorname{vol}\left(B_{n_{j}}(x)\right)$, hence these cubes are at a distance at least $(1-2 \varepsilon) \delta_{n_{j}}$ from $x$.

We know that any of them has to contain $2^{l+1}$ of vertices of $B_{n_{j}}(x)$ because the sides of those cubes are parallel to those of $B_{n_{j}}(x)$. We take $\varepsilon$ very small.

We now start a geometric part of the proof of Lemma 3.3. A pair $(A, B)$ where $A$ is a $d$-dimensional cube and $B$ is the difference of $A$ and a union of some $d$-dimensional cubes $C_{i}$ with disjoint interiors and sides parallel to those of $A$ will be called a $(d, l)$-pair when the cubes $C_{i}$ contain at least $2^{l+1}$ vertices of $A$ each but not the centre of $A$.

Lemma 3.4. Let $(A, B)$ be a $(d, l)$-pair. Then $B$ contains an interval between the centre of $A$ and the centre of one of its $(d-1)$-dimensional sides.

P r o of. Assume that this is not true and any such interval, say $x a$, meets one of the cubes $C_{i}$, say $C$. Look at a two-dimensional section of $A$, passing through the centre $x$ of $A$ and parallel both to the interval $x a$ and to one of the edges of $A$, contained in $C$. This section is a square for which every interval from the centre to the centres of sides meets one of the squares $C_{i}$ (we denote the squares in the same way as the cubes they come from). But $C$ contains two vertices of the square, so there are at most three squares $C_{i}$ as each of them contains at least one vertex. One square $C_{i}$ can meet only one interval - but there are four intervals, which leads us to a contradiction.

Lemma 3.5. Let $(A, B)$ be a $(d, l)$-pair. Then $B$ contains an $(l+1)$ dimensional cube $D$ whose edges are parallel to those of $A$ and have length greater than $c|A|$ and which lies in a cube $A^{\prime}$ with the same centre and orientation of sides as $A$ but with volume half that of $A$.

Proof. From the previous lemma we know that one of the intervals connecting the centre of $A$ with the centres of its sides lies completely in $B$; call it $D_{1}$. For $l=0$ the lemma follows immediately. For $l>0$ we look at the $(d-1)$-dimensional sections of $A$, orthogonal to $D_{1}$. We call two such sections similar if they are equal up to a translation. There are at most $2^{d-l-1}$ similarity classes of sections because they depend only on which of the cubes $C_{i}$ the section hyperplane meets. Hence there is a subinterval $D_{1}^{\prime} \subset D_{1}$ of length $c \cdot|A|$, lying in $A^{\prime}$, such that the sections passing through any two of its points are similar. 
Any of such sections is a $(d-1, l-1)$-pair because the intersection of a hyperplane $\pi$ and one of the cubes $C_{i}$ is a $(d-1)$-dimensional cube containing $2^{l}$ vertices of the cube $A \cap \pi$, and the centre of $A \cap \pi$ (the intersection of $D_{1}$ with the section plane) lies in $B \cap \pi$. The lemma follows by induction: we can now find an $l$-dimensional cube $D^{\prime}$ of side length comparable with $|A|$, contained in $B \cap \pi$ and the cube we seek is $D=D_{1}^{\prime} \times D^{\prime}$. See Figure 3 .

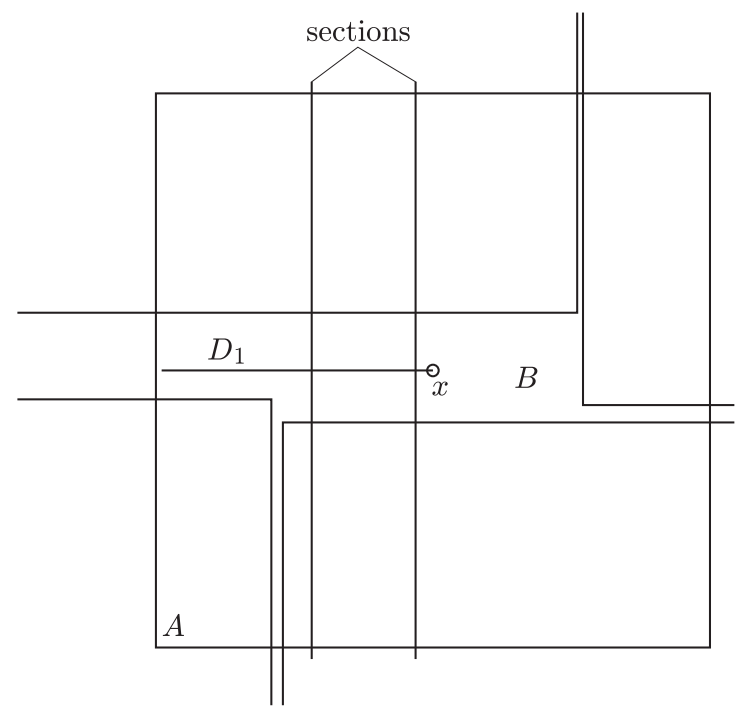

Fig. 3. A $(d, l)$-pair

To end the proof of Lemma 3.3 we need only see that $\left(B_{n_{j}}(x),\left(K_{n_{j}} \cap\right.\right.$ $\left.\left.B_{n_{j}}(x)\right)\right)$ is a $(d, l)$-pair if we add to $K_{n_{j}}$ all those cubes $E_{i}$ which contain less than $2^{l+1}$ vertices of $B_{n_{j}}(x)$. Then we have the cube $D(x)$ as in Lemma 3.5and it meets none of the cubes added for they are too far away from $x$.

4. General polyhedra. In this section we allow $\left(E_{i}\right)$ be any family of uniformly regular convex polyhedra with long edges. Let us start from some properties of such sets.

LEMma 4.1. Let $\left(E_{i}\right)$ be any family of uniformly regular convex polyhedra with long edges. Then

(i) the volume of $E_{i}$ is approximately equal to the dth power of its diameter, with uniform equivalence constants,

(ii) all the angles of $E_{i}$ are uniformly bounded away from zero, where by angles of a polyhedron we mean: in dimension 2 (for a polygon) its angles, in dimension 3 its solid angles and angles of its sides, and so on in higher dimensions, 
(iii) for any $l<d$ the number of $l$-dimensional sides of $E_{i}$ is uniformly bounded from above,

(iv) for any $l<d$ we can (as in the proofs of Lemmas 3.1 and 3.3) divide $E_{i}$ into (an infinite number of) subsets $E_{k}^{\prime}$. These subsets can be chosen to be uniformly regular polyhedra (with regularity constant independent of $l$ and $i)$, to form a Whitney partition for the union of l-dimensional sides of $E_{i}$ (more precisely: part of such a partition, contained in $E_{i}$ ) and to satisfy inequality (4) for all $t \in(l, l+1]$ with constant $c(t)$ independent of $i$.

Proof. Part (i) follows immediately from the very definition of uniformly regular sets. To prove (ii) one has to use convexity of the polyhedra, for if in a convex polyhedron an angle were very small then any ball contained in this polyhedron (in particular, in the angle) would be very small when compared with the diameter of the polyhedron.

To prove (iii) consider the radial projection of the boundary of $E_{i}$ on the maximal sphere contained in $E_{i}$. All the $(d-1)$-dimensional sides of $E_{i}$ are projected onto spherical polygons with $(d-1)$-dimensional volume bounded away from zero, hence the number of those sides is uniformly bounded from above - this gives our assertion in the case $l=d-1$. For $l$ smaller we proceed by induction, since all those $(d-1)$-dimensional sides are again a regular convex polyhedron with long edges (with a worse constant, but we repeat this induction step no more than $d$ times).

Part (iv) will be proven indirectly. It is easy to see that we can divide any of the sets $E_{i}$ into smaller uniformly regular polyhedra that will form a Whitney partition of the union of its $l$-dimensional sides. Inequality (4) holds for any such partition, the question is only if the constant $c(t)$ can be chosen independent of $i$.

Assume that this is not the case, and that for some $t>l$ one can find a sequence $\left(E_{j}\right)$ of uniformly regular convex polyhedra with long edges (without loss of generality, let $\left|E_{j}\right|=1$ ) and a partition $\left(E_{j k}^{\prime}\right) \subset E_{j}$ such that $c_{j}(t)>2^{t j}$, where $c_{j}$ is the constant in (4) for $\left(E_{j k}^{\prime}\right)$ and $E_{k}$.

We can see that the $l$-dimensional volume of the union of $l$-dimensional sides of $E_{j}$ is uniformly bounded by (iii). Consider a family of sets $F_{j}$, where each $F_{j}$ is a $2^{j}$ times smaller copy of $E_{j}$, translated in such a way as to contain the point $x_{j}=\left(2^{1-j}, 0, \ldots, 0\right)$. Denote by $F$ the closure of the union of all $l$-dimensional sides of the sets $F_{j}$. It is a compact set with Minkowski dimension $l$.

In each $F_{j}$ we have a Whitney partition $\left(F_{j k}^{\prime}\right)$ with respect to $F$. We have to add some additional sets to get a Whitney partition in the entire space, but this is easy because the sets $F_{j}$ are convex and far apart. We can estimate the convergence exponent of this partition. By Lemma 2.2 it is $l$; 
in our case, however, it is not smaller than $t$ because

$$
\sum_{j} \sum_{F_{j k}^{\prime} \subset F_{j}}\left|F_{j k}^{\prime}\right|^{t}=\sum_{j} \sum_{E_{j k}^{\prime} \subset E_{j}} 2^{-t j} \cdot\left|E_{j k}^{\prime}\right|^{t} \geq \sum_{j} 2^{-t j} c_{j}\left|E_{i}\right|^{t}=\infty .
$$

This contradiction ends the proof.

Now we can prove the analogues of Lemmas 3.1 and 3.2 for our more general families $\left(E_{i}\right)$. We skip the proofs, as they are exactly the same as the original proofs in the previous section, we only have to use Lemma 4.1 to validate the construction.

Lemma 3.3 is more difficult, though. We will alter slightly not only the proof, but the formulation as well. Let us start from the geometrical construction of a set we will call a generalized cube.

A one-dimensional generalized cube of size $a$ is just an interval of that length. This is the first step in the construction. In the second step, we look at the family of parallel $(d-1)$-dimensional hyperplanes, cutting the interval at an angle bounded away from zero. In each of these planes we choose an interval of length $a$ with one endpoint lying on the initial interval. We demand that the directions of these intervals should be piecewise the same when we move along the initial interval. The union of all those second order intervals is our generalized square. This set is the union of a finite number of parallelograms, one of whose sides add up to the initial interval (imagine some flags, waving on a common pole).

In the third step we have a family of second order intervals, each lying in an $(l-1)$-dimensional hyperplane. For each interval we take parallel $(d-2)$-dimensional hyperplanes, cutting the interval at an angle bounded away from zero, and we choose third order intervals (all of length $a$ ) in these hyperplanes, with an endpoint in a second order interval. We demand that the third order intervals have piecewise constant direction not only when we move along the second order interval, but also when we move along the first order interval (so the set we get is the union of a finite number of three-dimensional parallelepipeds); this restrics our freedom of choosing the $(d-2)$-dimensional hyperplanes. The union of all third order intervals will be called a generalized three-dimensional cube (it is difficult to visualize for one needs at least $\mathbb{R}^{4}$ to construct it).

We proceed to higher dimensions in the same way. A generalized $k$ dimensional cube of size $a$ has $k$-dimensional volume approximately $a^{k}$. We also have a well defined projection of part of the space $\mathbb{R}^{d}$ on the cube. Namely, after the last ( $k$ th) step of the construction we have a family of disjoint $(d-k+1)$-dimensional hyperplanes that cover the generalized cube and part of the space around it. The intersection of any of those planes with the generalized cube is an interval (a $k$ th order interval in our construction). 
For any point lying on one of those planes we can define the orthogonal projection on the line containing this interval.

Just as for an ordinary cube, the set of points whose projections lie on the generalized cube and which lie not farther than at distance $r$ from it has volume approximately $a^{k} r^{d-k}$. Also as for an ordinary cube, the $k$-dimensional volume of a projection of a ball of radius $r$ is not greater than $c r^{k}$.

We now denote by $B_{n_{j}}(x)$ a ball (not a cube) with centre $x$ and radius $\delta_{n_{j}}$. Our new version of Lemma 3.3 is as follows:

LEMMA 4.2. If the convergence exponent for $\left(E_{i}\right)$ is not equal to the upper Minkowski dimension of $\Lambda$ then there exists $C$ such that for any $\varepsilon$ and any $n_{j}$ sufficiently large, at least $\frac{1}{2} \sharp M_{n}$ of the sets $B_{n_{j}}(x) \cap K_{n_{j}}$ have volume smaller than $\varepsilon \delta_{n_{j}}^{d}$ and each of these contains a generalized $(l+1)$-dimensional cube $D(x)$ of size $C \cdot \delta_{n_{j}}$ at a distance at least $C \cdot \delta_{n_{j}}$ from the boundary of the ball $B_{n_{j}}(x)$.

Before we start the proof, notice that from this lemma Theorem 1.1 follows in precisely the same way as in the previous section from Lemma 3.3 (for cubes). The only properties of an $(l+1)$-dimensional cube we have used were:

- its $(l+1)$-dimensional volume is approximately equal to the $(l+1)$ th power of its side length,

- the projection of a ball of radius $r$ has $(l+1)$-dimensional volume not greater than $\mathrm{cr}^{l+1}$,

and both these properties hold for generalized cubes as well. Also the change in the sets $B_{n_{j}}(x)$ (balls instead of cubes) is irrelevant. Hence we need only prove Lemma 4.2 .

We rewrite the proof of Lemma 3.3, in fact. As before, we can show (using Lemma 4.1(iii)) that for any $\varepsilon$ and $n_{j}$ large enough for most of the points in $M_{n_{j}}$ only a small part (of volume $\varepsilon \delta_{n_{j}}^{d}$ ) of the ball $B_{n_{j}}(x)$ is covered by $K_{n_{j}}$; the rest is covered by some big polyhedra $E_{i}$ whose $l$-dimensional sides lie far away from $B_{n_{j}}(x)$. These polyhedra avoid $x$.

If these polyhedra are big enough (when $\delta$ was chosen small enough) then the intersection of any of them with a hyperplane of codimension one passing through $B_{n_{j}}(x)$ chosen in such a way that the angles between this hyperplane and all the sides of the polyhedron are bounded away from zero - even when the bounds are not very strong - is again a regular convex polyhedron with long edges in $\mathbb{R}^{d-1}$, and the $(l-1)$-dimensional sides of this polyhedron lie again far away from the intersection of this hyperplane with $B_{n_{j}}(x)$. Of course, such an operation makes all the constants (especially the one in the definition of long edges) worse, but we will use it only a finite number of times (at most $d$ ), so we can control how bad they become and neutralize this by the choice of $\delta$. 
We can repeat this procedure. In particular, by choosing $\delta$ small enough we can arrange that for given bounds on the angles between a two-dimensional plane and the angles of the polyhedron the edges of the resulting intersection (which is a polygon) are greater than $2 \delta_{n_{j}}$, hence only one of its vertices can lie inside $B_{n_{j}}(x)$.

As before, we let a generalized $(d, l)$-pair be a pair $(A, B)$ where $A$ is the ball in $\mathbb{R}^{d}$ and $B$ is the difference of $A$ and some family of big $d$-dimensional uniformly regular convex polyhedra with long edges whose $l$-dimensional sides lie far away from $A$ and which do not contain the centre of $A$. We can estimate the number of those polyhedra from above, because their angles are bounded from below (Lemma 4.1(ii)).

We now generalize Lemmas 3.4 and 3.5 to our present situation.

Lemma 4.3. For any generalized $(d, l)$-pair $(A, B)$ if the removed polyhedra are big enough then one of the radii of the ball $A$ lies in $B$.

Proof. We can find a two-dimensional plane that passes through $x$ and whose intersection with one of the big polyhedra has no vertices inside $A$. We have some freedom in choosing such a plane, hence we can get lower bounds on the angles between this plane and the sides of all other big polyhedra (as there are only a bounded number of them). Hence the intersections of the plane with the other polyhedra will have at most one vertex inside $A$, and the rest far away.

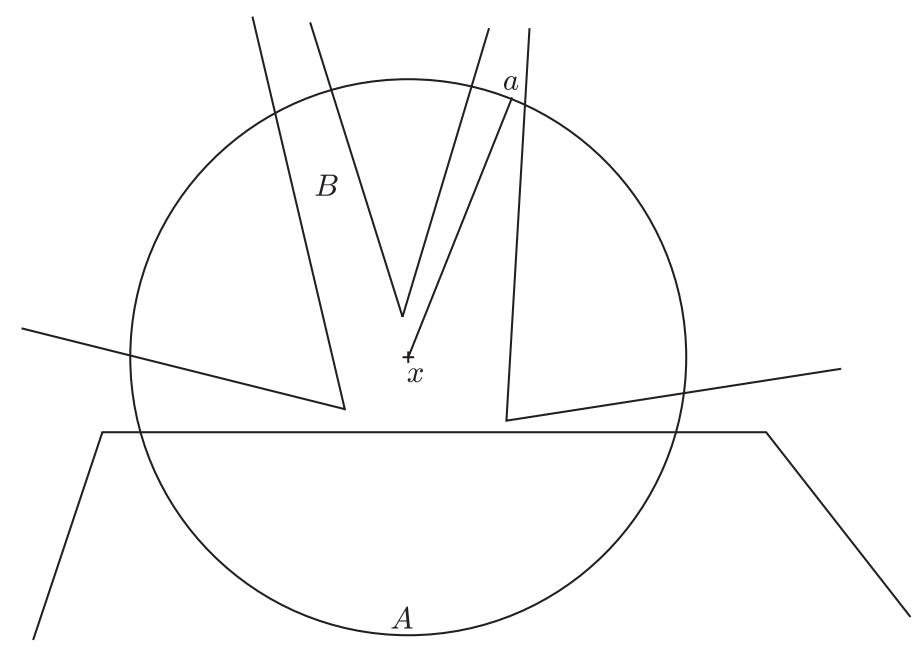

Fig. 4. Two-dimensional section of a generalized $(d, l)$-pair

We call an edge of one of those big polygons (intersections of the big polyhedra with the plane) visible from $x$ if the intervals connecting $x$ to the part of this edge contained in $A$ do not meet the interior of the polygon. 
Among the big polygons that meet $A$, there is one that has only one (visible) edge meeting $A$ and each of the remaining polygons has at most two edges meeting $A$, at least one of them visible. If two neighbouring edges of two neighbouring polygons are visible - which must happen somewhere, see Figure 4 - one can find a radius of $A$ passing between them, hence contained in $B$.

Lemma 4.4. For any generalized $(d, l)$-pair $(A, B)$ the set $B$ contains a generalized $(l+1)$-dimensional cube of size comparable with the radius of $A$.

Proof. From Lemma 4.3, we can find in $B$ some radius of $A$; denote it by $x a$.

Because the number of removed polyhedra is bounded, we can find a family of parallel $(d-1)$-dimensional hyperplanes with the some lower bounds on their angles with the sides of the removed polyhedra and with the interval $x a$. Take only those hyperplanes that cut the interval $x a$ inside $\frac{1}{2} A$ (that is, the ball of the same centre but half its radius).

Choose one such plane $\pi$. The intersection of $\pi$ with $A$ is a $(d-1)$ dimensional ball of almost the same radius. Its centre does not lie in $\pi \cap x a$, but we can find inside $\pi \cap A$ a ball $A^{\prime}$ with centre in $\pi \cap x a$ and a slightly smaller radius. The intersection of $\pi$ with each big polyhedron is an $(l-1)$ dimensional regular convex polyhedron with long edges (because the angles between $\pi$ and the sides of the big polyhedra are bounded from below) and it does not contain the centre of $A^{\prime}$.

Hence, $\left(A^{\prime}, \pi \cap B\right)$ is a $(d-1, l-1)$-pair. By Lemma 4.3 we can find a radius of $A^{\prime}$ contained in $\pi \cap B$, hence in $B$. Let us repeat this for all the hyperplanes, choosing one interval in each of them. If an interval does not intersect a polyhedron then parallel intervals close enough will not intersect it either. This way (for planes $\pi^{\prime}$ close enough to $\pi$ ) we can choose those intervals to be parallel to the one chosen for $\pi$. Hence we may choose all those intervals to have only a finite number of directions.

As we have constructed a generalized square (in fact, a rectangle; we still have to shorten some of its "sides" to get a generalized square), we proceed by induction.

Using this lemma for the pair $\left(B_{n_{j}}(x), K_{n_{j}} \cap B_{n_{j}}(x)\right)$ we get the assertion of Lemma 4.2, hence of Theorem 1.1.

\section{References}

[1] C. Bishop, Minkowski dimension and the Poincaré exponent, Michigan Math. J. 43 (1996), 231-246.

[2] —, Geometric exponents and Kleinian groups, Invent. Math. 127 (1997), 33-50. 
[3] L. Carleson, P. W. Jones and J. C. Yoccoz, Julia and John, Bol. Soc. Brasil. Mat. 25 (1994), 1-30.

[4] K. Falconer, Fractal Geometry. Mathematical Foundations and Applications, Wiley, Chichester, 1990.

[5] O. Martio and M. Vuorinen, Whitney cubes, p-capacity and Minkowski content, Exposition. Math. 5 (1987), 17-40.

[6] P. Mattila, Geometry of Sets and Measures in Euclidean Spaces, Cambridge Univ. Press, Cambridge, 1995.

[7] P. J. Nicholls, The Ergodic Theory of Discrete Groups, Cambridge Univ. Press, Cambridge, 1989.

[8] C. Pommerenke, Boundary Behaviour of Conformal Maps, Springer, Heidelberg, 1992.

[9] M. Rams, Box dimension and self-intersecting Cantor sets, doctoral thesis, IM PAN, 1999 (in Polish).

[10] E. Stein, Singular Integrals and Differentiability Properties of Functions, Princeton Univ. Press, Princeton, 1970.

[11] C. Tricot, Porous surfaces, Constr. Approx. 5 (1989), 117-136.

[12] - Curves and Fractal Dimension, Springer, Berlin, 1995.

[13] - Mesures et dimensions, doctoral thesis, Univ. Paris-Sud, 1983.

Institute of Mathematics

Polish Academy of Sciences

Śniadeckich 8

00-950 Warszawa, Poland

E-mail: rams@snowman.impan.gov.pl

Received 4 June 1998;

in revised form 6 July 1999 and 19 March 2000 